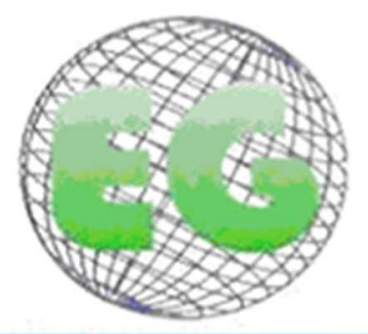

ISSN 1695-6141 $\mathbf{N}^{\circ} 63$

\title{
REVISIONES
}

\section{Revisión bibliográfica sobre la memoria histórica de pandemias anteriores en revisiones de enfermería sobre COVID-19: una realidad secularmente documentada}

Bibliographic review on the historical memory of previous pandemics in nursing reviews on COVID-19: a secularly documented reality

\author{
Joaquín León Molina ${ }^{1}$ \\ M. Fuensanta Hellín Gil ${ }^{1}$ \\ Eva Abad Corpa ${ }^{2}$
}

\begin{abstract}
1 Enfermera Hospital Clínico Universitario Virgen de la Arrixaca de Murcia; Grupo Cuidados Enfermeros Avanzados del Instituto Murciano de Investigación Biosanitaria Arrixaca. Murcia. España.

2 Enfermera vinculada Hospital General Universitario Reina Sofía de Murcia; Profesora Contratada Doctora vinculada Universidad de Murcia. Grupo Cuidados Enfermeros Avanzados del Instituto Murciano de Investigación Biosanitaria Arrixaca. Murcia. España.
\end{abstract}

\section{https://doi.org/10.6018/eglobal.456511}

Recibido: $17 / 11 / 2020$

Aceptado: 10/01/20201

\section{RESUMEN:}

Introducción: Las revisiones sobre COVID-19 deberían reflejar antecedentes históricos de anteriores pandemias.

Objetivo: Nos planteamos mapear el contenido de revisiones recientes, en el área de enfermería sobre COVID-19, para comprobar si se hace alusión a crisis sanitarias por epidemias y enfermedades infecciosas.

Metodología. Revisión narrativa descriptiva. Se consultaron la Web de la Ciencia, PubMed y Lilacs para identificar las revisiones; se consultó el contenido de los documentos para detectar la presencia de descriptores y términos relativos a las pandemias anteriores al siglo XXI de la humanidad, atendiendo a los criterios de inclusión y objetivos del estudio.

Resultados: Sólo en 11 documentos de los 192 identificados se localizaron reseñas pertinentes.

Conclusiones: Pueden existir reticencias a la hora de utilizar documentación publicada hace más de un siglo; sin embargo, sería aconsejable no perder la memoria histórica de crisis pandémicas que ha padecido la humanidad durante milenios.

Palabras clave: Pandemias; Epidemias; Historia; Historia Antigua.

ABSTRAC:

Introduction: Reviews for COVID-19 should reflect historical antecedents from previous pandemics. Objective: We plan to map the content of recent reviews in the nursing area on COVID-19 to see if it refers to health crises due to epidemics and infectious diseases.

Methodology: Descriptive narrative review. The Science Web, PubMed and Lilacs were consulted to identify the reviews; The content of the documents was consulted to detect the presence of descriptors 
and terms related to pandemics prior to the 21st century of humanity, taking into account the inclusion criteria and objectives of the study.

Results: Relevant reviews were found only in 11 documents of the 192 identified.

Conclusions: There may be reluctance to use documentation published more than a century ago; However, it would be advisable not to lose the historical memory of pandemic crises that humanity has suffered for millennia

Key words: Pandemics; Epidemics; History; Ancient History.

\section{INTRODUCCIÓN}

Desde la aparición del Coronavirus responsable de la COVID-19 a finales del año 2019, y posteriormente desde la declaración de la enfermedad como pandemia en marzo de 2020, nunca antes se había producido un incremento de publicaciones en revistas indexadas en las principales bases de datos bibliográficas (BDB) en apenas 12 meses. Estas BDB han dejado libre acceso para investigadores y profesionales sanitarios. Ahora bien, cuando escribimos sobre crisis sanitarias deberíamos, razonablemente, documentar los argumentos sin olvidar la bibliografía histórica que avalan que epidemias y pandemias han existido secularmente. Documentos en todo tipo de soporte sobre peste, tifus (tabardillo), tosferina, sarampión, cólera, gripe, Sida (HIV), meningitis, tuberculosis, pueden hacernos recordar que la situación creada por la COVID-19 no es nueva. Las enfermedades infecciosas en forma de epidemia o pandemia, han acompañado a la humanidad a lo largo de su historia.

El uso correcto de la terminología es, también, importante. Para consultar el significado de, entre otros conceptos, epidemiologia, peste, epidemia, pandemia, endemia, brote epidemiológico, zoonosis, lazareto podemos utilizar el diccionario de términos médicos de la Real Academia Nacional de la Medicina de España. ${ }^{(1)}$

Un primer paso sería detectar las pandemias más antiguas de la humanidad y aquellas que han causado mayor número de muertes. En la tabla siguiente, de elaboración propia basada en muy diversa bibliografía y fuentes, se enumeran las epidemias y pandemias más antiguas y más mortíferas. 


\begin{tabular}{|c|c|c|}
\hline \multicolumn{3}{|c|}{ Epidemias y pandemias más importantes } \\
\hline Clasificadas & Años & Denominación \\
\hline \multirow{5}{*}{ Epidemias y pandemias más antiguas } & $1200 \mathrm{aC}$ & Influenza \\
\hline & $429 \mathrm{aC}$ & Plaga de Atenas \\
\hline & $412 \mathrm{aC}$ & Epidemia de Atenas \\
\hline & $165 d C$ & Peste Antoniana \\
\hline & $250 \mathrm{dC}$ & Peste Cipriana \\
\hline \multirow{12}{*}{$\begin{array}{l}\text { Epidemias y pandemias más } \\
\text { mortíferas (más de } 1 \text { millón de } \\
\text { muertes, ordenadas por mayor } \\
\text { mortalidad) }\end{array}$} & 1346 & Peste Negra \\
\hline & 1918 & Gripe Española \\
\hline & Desde 1981 & SIDA \\
\hline & 541 & Plaga Justiniano \\
\hline & 1545 y 1576 & Epidemias de Cocolitzi \\
\hline & 1520 & Epidemia de viruela de Tecnochtitlan \\
\hline & 165 & Peste Antonina \\
\hline & 1772 & Peste Persa \\
\hline & 1656 & Peste de Nápoles \\
\hline & 1957 & Gripe asiática \\
\hline & 1968 & Gripe de Hong Kong \\
\hline & Desde 2019 & COVID-19 \\
\hline
\end{tabular}

\section{Las epidemias en el arte}

Por mera cuestión de extensión y por la temática de esta revista, sólo se han citado algunas muestras en literatura y pintura, sin olvidar que en arquitectura, escultura, música, danza y cine existen otros ejemplos.

En literatura encontramos referencias a pandemias y epidemias, generalmente peste, en diferentes textos clásicos y de la época moderna. En el siglo $\mathrm{V}$ antes de Cristo Tucídides, padre de la historiografía científica y Sófocles hicieron referencias, en sus obras, a la epidemia de peste que asolo Atenas ${ }^{(2)}$. El Decamerón, escrito en 1352 por Boccaccio, comienza con una descripción de la peste bubónica que golpeó Florencia en 1348 y cómo un grupo de diez jóvenes que huyen de la plaga, se refugian en una villa en las afueras de Florencia(3-5). Las enfermedades que asolan Londres durante el siglo XVI se reflejan en el teatro de Shakespeare; En Romeo y Julieta la epidemia de peste y la cuarentena de Verona juega un papel fundamental(6). Daniel Defoe en Diario del año de la peste que relata la Gran Plaga en Londres (1665) describe situaciones que hoy nos parecían actuales por su total similitud ${ }^{(7)}$. La peste, de Albert Camus, publicada en 1947, cuenta la historia de unos doctores que descubren el sentido de la solidaridad en su labor humanitaria en la ciudad de Orán mientras era azotada por una epidemia de peste; el texto comienza con una cita del anteriormente citado Defoe: "Tan razonable como representar una prisión de cierto género por otra diferente es representar algo qué existe realmente por algo que no existe"(7).

En pintura existen numerosas muestras de los signos de las enfermedades infecciosas (peste, lepra, tuberculosis y sífilis, etc.) y el temor que causaron en la población y sus implicaciones socioculturales. Los cuatro jinetes del apocalipsis de Durero en 1498; San Roque entre las víctimas de la peste y la Virgen de la Gloria de Ponte Basano en 1575; La peste de Asdod de Poussin en 1630; Peste de Atenas de 
Sweerts en 1652; La plaza del mercado de Nápoles durante la peste de Gargiulo en 1656 y Corral de apestados de Goya en $1798^{(8)}$ son algunos ejemplos

\section{Textos de medicina sobre epidemias y pandemias (peste)}

Historiadores médicos como López Piñero(9) o Sánchez Granjel(10), entre otros, incluyen en sus obras autores en cuyos textos se esconden títulos y capítulos que tratan de peste y epidemias.

Hasta el Siglo XVI podemos destacar: El papiro de Ebers que menciona unas fiebres pestilentes que asolaron a la población de las márgenes del Nilo alrededor del año 2000 a.C; probablemente el texto en el que se hace la más antigua referencia a un padecimiento colectivo. Abu Ghiaphar Ibn Khatemar, médico árabe natural de Almería, describió la epidemia que azotó Almería en 1347. Abu Mohamed Ben Alkhathib, médico árabe natural de Granada, escribió en 1348 sobre sobre la peste que atacó esta ciudad; Guy Chauliac en 1363 escribió sobre el paso de la peste por Avignon y comentó cómo los padres no visitaban a sus hijos ni los hijos a los padres y cómo los médicos no se arriesgaban visitar a los enfermos por miedo a quedar infectados.

En el siglo XVI se combina el auge de la imprenta y el humanismo médico, suponiendo un punto de inflexión en la difusión del libro en general y del libro de materia médica en particular. Destacamos, entre cientos de textos recuperables en bibliotecas digitales como Dioscórides de la Universidad Complutense o Cervantes Virtual, autores que hacen referencia en sus textos a la peste, bubones a su prevención y tratamiento, a efectos colaterales en la sociedad y en la psicología. Basta con teclear en sus formularios de búsqueda el término peste, para el periodo de años 1501 a 1600 y en autores para identificar títulos como Regiment preservatiu e curatiu de la pestilencia escrito por Alcanys en 1490, Tratado util e muy provechoso contra toda pestilencia e ayre corupto de Fores escrito en 1507, Libro Información y curación de la peste de Zaragoza, y Preservación contra la peste en general de Porcell escrito en 1565, Remedios preservativos y curativos para el tiempo de la peste y otras curiosas experiencias escrito por Martínez de Leiva en 1597, De natura et conditionibus, praservatione et curatione pestis de Luis Mercado en 1598.

\section{Lazaretos y otros recintos de aislamiento}

Establecimientos hoy en desuso nos recuerdan que el aislamiento de personas infectadas en edificios apartados ha existido durante toda la historia. No son las actuales "Arcas de Noé" o "Grupos Burbuja" o los hospitales COVID, pero podríamos encontrar similitudes.

Hasta finales del Siglo XIX la navegación marítima constituyó uno de los principales mecanismos de difusión de enfermedades infecciosas y un sistema de expansión de epidemias y pandemias ${ }^{(11)}$. Los pasajeros de algunos barcos sospechosos de portar enfermedades contagiosas eran confinados en edificios para cuarentena. Los lazaretos eran establecimientos sanitarios para el aislamiento, desinfección y asistencia de personas o animales infectados o sospechosos de padecer una enfermedad epidémica o contagiosa; eran estaciones de cuarentena ${ }^{(1,11,12)}$. Artículos 
sobre la historia de lazaretos concretos, como el de Mahón, nos recuerdan la existencia de estos edificios de confinamiento(13).

Otros recintos de aislamiento sanitario, confinamiento o aislamiento de infectados son las leproserías (establecimiento, casa o poblado sanitario destinado específicamente a prestar asistencia sanitaria a los enfermos de lepra) ${ }^{(1)}$ y los sanatorios antituberculosos (generalmente en zonas de sierra) como el de Sierra Espuña en Murcia, el Real Sanatorio de Guadarrama en Madrid, el Dispensario Antituberculoso del Raval de Barcelona. El documento de la escuela de sanidad del Instituto Carlos III sobre arquitectura sanitaria y sanatorios tuberculosos ${ }^{(14)}$ recopila información detallada de estos edificios en España y en Europa.

\section{Sobre gripe española}

La pandemia de Influenza de 1917, mal llamada Gripe Española, es la crisis sanitaria de la historia más reciente con mayores similitudes sanitarias y socio económicas con la actual COVID-19. Sobre ella se publicaron en su momento artículos en las principales revistas médicas (The British Medical Journal y The Journal of the American Medical Association). Sus autores ya preveían pandemias futuras ${ }^{(15)}$. Alfonso Mendiola en Crisis e historia o pandemia y operación historiográfica ${ }^{(16)}$ hace una oportuna reflexión filosófica e histórica de la contextualización y/o descontextualización de la actual crisis de la COVID-19.

En The great influenza. The epic story of the deadliest plague in history ${ }^{(17)}$, el autor narra la historia épica de la plaga más mortífera de la historia. El libro, bien documentado, está destinado a médicos, científicos, estudiantes de salud, historiadores ya que se combinan temas sanitarios con ciencia y política. De escritura comprensible y convincente es un libro con mucha relevancia para los tiempos actuales de la COVID-19(18).

Gould(19) también revisa el texto de Barry sobre la pandemia de influenza y destaca, igualmente, cómo el libro se narra desde la perspectiva de los científicos y líderes políticos. Se narran historias personales, de intuiciones, médicos y científicos que combatieron la enfermedad con ciencia y tenacidad. Describe los 2 caminos usados en ese momento: explorar la epidemiología y rastrear pistas en el laboratorio.

Para Wei(20) la enfermedad por coronavirus 2019 es la mayor crisis de salud pública desde la pandemia de influenza de 1918 y presenta, como entonces, una amenaza mundial sin precedentes.

\section{OBJETIVO}

Mapear la producción de conocimientos sobre historia y antecedentes de pandemias; comprobar si las revisiones bibliográficas en el área de enfermería sobre pandemias, y especialmente COVID-19, se hacen eco de antecedentes históricos, específicamente a episodios anteriores al siglo XXI. 


\section{METODOLOGÍA}

Se ha realizado una revisión bibliográfica narrativa y descriptiva. Para el marco histórico y conceptual, se han consultado bibliotecas online, digitales o virtuales, buscando en ellas como descriptores los términos peste y epidemia; se consultó el Diccionario online de términos médicos de la Real Academia Nacional de Medicina de España.

Para la revisión, se consultaron la Web de la Ciencias (WOS), PubMed y Lilacs con los términos COVID, Coronavirus y pandemia (pandemic) para revisiones publicadas desde 2020 en el área de enfermería. Los criterios de inclusión fueron revisiones bibliográficas en el área de enfermería y publicadas desde 2020 sobre pandemias en general y COVID-19 en particular, así como otra bibliografía fuera de esta temporalidad, relevante para el tema objeto de estudio. Se excluyeron documentos en japonés, alemán, danés o sueco; los que, a pesar de tratar de materias pandémicas o COVID-19, no hacían mención a antecedentes históricos a pandemias anteriores al siglo XXI. Un documento fue excluido por la imposibilidad de recuperación. Para la recuperación de documentos se han utilizado la WOS, la Biblioteca Virtual de Murciasalud y Google Scholar (Ver tabla 2: flow diagram). Se utilizó el gestor bibliográfico EndNote $x 7$ licencia personal y EndNote Web a través de la Universidad de Murcia.

Tabla 2: Diagrama de flujo del estudio

\begin{tabular}{|c|c|c|c|}
\hline $\begin{array}{l}\text { Tema: Coronavirus, Covid, } \\
\text { Pandemia Pandemic }\end{array}$ & & Bases de datos: & \\
\hline Tipo documento: Revisiones & & $\begin{array}{l}\text { WOS (WOS, CCC,DIIDW, KJD, } \\
\text { MEDLINE, RSCI, SCIELO) }\end{array}$ & 56 \\
\hline $\begin{array}{l}\text { Área investigación: Revistas } \\
\text { Enfermería }\end{array}$ & & PubMed & 32 \\
\hline Periodo: año 2020 & & Lilacs & 126 \\
\hline Exclusiones & & Identificaciones & 214 \\
\hline $\begin{array}{l}\text { Idioma (alemán, japonés, } \\
\text { eslavo) }\end{array}$ & 4 & Duplicados & 22 \\
\hline Menor pertinencia según título & 92 & & 192 \\
\hline No disponible & 1 & & \\
\hline $\begin{array}{l}\text { Tras primera fase de lectura pre- } \\
\text { crítica no hacen referencia a }\end{array}$ & 84 & & \\
\hline & 181 & Documentos revisión & 13 \\
\hline & & $\begin{array}{l}\text { Documentos introducción y } \\
\text { documentación }\end{array}$ & 17 \\
\hline & & Referencias en bibliografía & 30 \\
\hline
\end{tabular}




\section{RESULTADOS}

En la investigación histórica se identificó abundante y suficiente material bibliográfico con que basar los antecedentes más remotos de una pandemia. Resultó complicado filtrar tan abundante información, por lo que se sólo se ha reflejado en la introducción hilos documentales para un estudio más profundo sobre pandemias en la humanidad.

En el estudio bibliográfico se identificaron 214 revisiones de las que 22 estaban duplicadas. Se excluyeron por idioma, pertinencia y disponibilidad 185; se analizaron 11 revisiones tratando de identificar las menciones a episodios pandémicos anteriores o a consecuencias de anteriores crisis por epidemias y pandemias.

\section{Atendiendo al origen de la pandemia}

Según Nagib(21) constantemente estamos invadiendo los espacios de los demás animales vivos. Eso no es noticia. Olvidamos la historia de otras enfermedades que surgieron de animales que fueron domesticados en el Neolítico, hace más de 10.000 años. La era de la agricultura surgió acompañada de la domesticación de animales salvajes.

\section{Atendiendo a los impactos en sistemas de salud}

Para Keller(22) el SARS-CoV-2 es un evento extraordinario en la historia de los sistemas de salud. Ha tenido un impacto en el mundo de una manera rápida, universal y poderosa, exponiendo deficiencias tanto en la resiliencia de los sistemas de salud como en la difusión de las mejores prácticas durante una situación en evolución.

Paterson ${ }^{(23)}$ destaca el papel fundamental de las enfermeras en la prevención de una mayor propagación de la influenza y la reducción de la gravedad de la pandemia que consideran la epidemia más devastadora de la historia.

Daniela Savi(24) nos recuerda a Nightingale en los desafíos actuales de enfermería en manejo en la pandemia COVID-19.

En el ya citado artículo de Gould(19) sobre el texto de Barry(17), se comenta el libro de Johnson S El mapa fantasma: la historia de la epidemia más aterradora de Londres ${ }^{(25)}$ en el que se narra el brote de cólera en el Londres Victoriano de 1851 que rápidamente se convirtió en una epidemia y cómo cambió la ciencia, las ciudades y el mundo moderno. Se crea un realista relato de la vida durante este tiempo mientras describe los esfuerzos de la comunidad médica para rastrear la enfermedad utilizando métodos científicos y un trabajo de detective persistente, un modelo que todavía sirve a los proveedores de salud pública de hoy. Se relata como un médico comprometido rastreó la enfermedad sin la disponibilidad de las herramientas epidemiológicas modernas.

En otro artículo Gould(26), que no ha sido posible consultar por no estar disponibles en los recursos utilizados, se analiza el aprendizaje y normas de cuidado en la COVID-19 


\section{Atendiendo a efectos colaterales}

Usher ${ }^{(27)}$ comenta que a lo largo de la historia, han causado enormes pérdidas de vidas $\mathrm{y}$, como resultado, han generado un sufrimiento social extremo.

Para Wei(20) la pandemias de la COVID-19 es la mayor crisis de salud pública desde la pandemia de influenza de 1918 y presenta una amenaza mundial sin precedentes.

\section{Otros documentos de interés}

Dado el escaso número de resultados pertinentes encontrados se propone la lectura de algunos documentos relevantes.

Mirzaie $^{(28)}$ realiza una revisión de la literatura narrativa sobre las opciones de la medicina tradicional para el tratamiento de la COVID-19.

Roney ${ }^{(29)}$ Describe la necesidad de analizar las secuelas de la COVID-19, con efectos sin precedentes en la historia, según avance y continúe en los años venideros.

Para Rosa $\mathrm{W}^{(30)}$, las enfermeras son líderes en la atención al paciente, educación y ciencia del cuidado y analiza cómo los factores ambientales, las características psicosociales y las relaciones interpersonales, influyen en el bienestar de las enfermeras y comenta cómo el entorno también contribuye a sus niveles de agotamiento y satisfacción laboral.

\section{CONCLUSIONES}

A pesar de que la temática de un artículo o revisión sea una epidemia o pandemia concreta, quizás sea pertinente reflejar hechos históricos y bibliografía antigua para tener conocimiento y constancia de que estas crisis sanitarias se han repetido a lo largo de la humanidad.

Por otra parte, es importante fundamentar una opinión. La pandemia actual no es una invención; hoy tenemos más evidencia científica que hace 3 décadas y, además, podemos comprobar que en la historia, la humanidad ya ha sufrido múltiples epidemias y pandemias. El olvido puede ser causa de clonaciones de hechos históricos.

Con la pertinencia y pertenencia de los documentos hemos comprobado que, una vez más, una crisis sanitaria en forma de pandemia esté asolando nuevamente a la humanidad.

Dado que en la mayoría de las revisiones mapeadas falta una perspectiva histórica mínima, abogamos por que artículos y revisiones bibliográficas sobre COVID-19 u otras pandemias, reflejen, o al menos mencionen, bibliografía referente a anteriores crisis pandémicas.

Si la evidencia científica es imprescindible en la actividad asistencial y en la investigación, la memoria o evidencia histórica es necesaria. 


\section{Agradecimientos}

A los profesionales sanitarios que han estado, están y estarán al frente de la batalla contra la 'peste'.

\section{REFERENCIAS}

1. Real Academia Nacional ME. Diccionario online de términos médicos de la real Academia Nacional de Medicina de España [visitado 12/10/2020]. Accesible en: http://dtme.ranm.es/index.aspx.

2. Saravia de Grossi MI. Edipo rey de Sófocles: Una lectura del Estásimo IV. Argos. 2014; 37(2): 119-40. Accesible en: https://www.scienceopen.com/document?vid=81f13d7b-c2cf-4531-a553-

\section{6f87b9ec3468}

3. Cuadrada $C$. The spread of the plague: A sciento-historiographic review. Med Hist (Barc). 2015; (2): 4-19. Accesible en: https://www.ncbi.nlm.nih.gov/pubmed/26399143

4. Lombardi A. THE DEVIL IN THE FLESH: A READING OF BOCCACCIO'S DECAMERON. Alea-Estudos Neolatinos. 2012; 14(2): 180-200. Accesible en: https://www.scielo.br/pdf/alea/v14n2/03.pdf

5. Wigand ME, Becker T, Steger F. Psychosocial Reactions to Plagues in the Cultural History of Medicine: A Medical Humanities Approach. J Nerv Ment Dis. 2020; 208(6): 443-4. Accesible en: https://www.ncbi.nlm.nih.gov/pubmed/32472811

6. Paya E. Las enfemedades infecciosas en la obra de Willian Shakespeare. Revista Chilena De Infectologia. 2013; 30(6): 660-. Accesible en: https://scielo.conicyt.cl/pdf/rci/v30n6/art14.pdf

7. Guerard A, Sr. Looking Back in the Pages of WLT: OUR 1948 REVIEW OF ALBERT CAMUS'S LA PESTE. World Literature Today. 2020; 94(3): 69-. Accesible en: https://doi.org/10.7588/worllitetoda.94.3.0069

8. Seoane Prado R. Las enfermedades infecciosas en el arte. Anales de la Real Academia Nacional de Medicina. 2018; 135(3). Accesible en: https://analesranm.es/wp-content/uploads/2018/numero 135 03/pdfs/ar135-rev12.pdf 9. López Piñero JM. Historia de la medicina universal. Valencia: Ayuntamiento de Valencia; 2010.

10. Sanchez Granjel L. Historia de la medicina española. Barcelona: Sayma; 1962. 205 p. p.

11. Ocaña Quevedo L. El Lazareto de Mahón. Medicina y Seguridad del Trabajo. 2007; 53(207): 63-9. Accesible en: http://scielo.isciii.es/pdf/mesetra/v53n207/original8.pdf

12. Rodríguez Toro JG, Pinto García R. La lepra. Imagenes y conceptos. Antioquia2007. $177 \mathrm{p}$.

13. Maldonado $\mathrm{H}$, Hernandez $\mathrm{M}$. Memorias de un sanatorio antituberculoso. Biomedica : revista del Instituto Nacional de Salud. 2004; 24 Supp 1: 27-33. Accesible en: http://www.scielo.org.co/pdf/bio/v24s1/v24sa05.pdf

14. Escuela Nacional S. Arquitectura sanitaria: sanatorios antituberculosos2014. Accesible

en: http://gesdoc.isciii.es/gesdoccontroller?action=download\&id=20/02/2015-5b9b4cb421. 15. Manrique-Abril FG, Beltrán-Morera J, Ospina-Díaz JM. Cien años después, recordando cómo BMJ y JAMA comunicaron la pandemia de gripe de 1918-1919. Revista de Salud Pública. 2018; 20(6): 787-91. Accesible en: http://www.scielo.org.co/pdf/rsap/v20n6/0124-0064-rsap-20-06-787.pdf 
16. Mendiola A. Crisis e historia o pandemia y operación historiográfica. Historia y grafía. 2020; (55): 173-5. Accesible en: http://www.scielo.org.mx/pdf/hg/n55/14050927-hg-55-173.pdf

17. Barry JM. The great influenza: the epic story of the deadliest plague in history. New York: Viking; 2004. 546 p p.

18. Palese $P$. The great influenza The epic story of the deadliest plague in history. The Journal Clinic Investigation. 2004; 114(2). Accesible en: https://doi.org/10.1172/JCl22439

19. Gould KA. The Great Influenza: The Story of the Deadliest Pandemic in History. Dimensions of Critical Care Nursing. 2020; 39(3): 165-6. Accesible en: https://doi.org/10.1172/JCl22439

20. Wei E, Segall J, Villanueva Y, et al. Coping With Trauma, Celebrating Life: Reinventing Patient And Staff Support During The COVID-19 Pandemic. Health Affairs. 2020; 39(9): 1597-600. Accesible en: https://doi.org/10.1377/hlthaff.2020.00929

21. Nagib Atallah A. COVID-19, evidências clínicas mais confiáveis. Os ensaios clínicos e as revisões sistemáticas. Daignostico y tratamiento. 2020; 25(2). Accesible en: $\quad$ http://docs.bvsalud.org/biblioref/2020/07/1115994/ok-rdt 252edit alvaroatallah.pdf

22. Keller KG, Reangsing C, Schneider JK. Clinical presentation and outcomes of hospitalized adults with COVID-19: A systematic review. Journal of Advanced Nursing. 2020. Accesible en: https://onlinelibrary.wiley.com/doi/epdf/10.1111/jan.14558

23. Paterson C, Gobel B, Gosselin T, et al. Oncology Nursing During a Pandemic: Critical Re flections in the Context of COVID-19. Seminars in Oncology Nursing. 2020; 36(3). Accesible en: https://doi.org/10.1016/j.soncn.2020.151028

24. Geremia DS, Vendruscolo C, Celuppi LC, et al. 200 Years of Florence and the challenges of nursing practices management in the COVID-19 pandemic. Revista Latino-Americana De Enfermagem. 2020; 28. Accesible en: https://www.scielo.br/scielo.php?pid=S0104-11692020000100403\&script=sci arttext

25. Johnson S. The ghost map : the story of London's most terrifying epidemic * and how it changed science, cities, and the modern world. New York: Riverhead Books; 2006. 299 p. p.

26. Gould KA. IHI Virtual Learning Hour Special Series: COVID-19 and Crisis Standards of Care. Dimensions of Critical Care Nursing. 2020; 39(6): 351-2. Accesible en: no disponible

27. Usher $\mathrm{K}$, Jackson D, Durkin J, et al. Pandemic-related behaviours and psychological outcomes; A rapid literature review to explain COVID-19 behaviours. Int J Ment Health Nurs. 2020. Accesible en: https://doi.org/10.1111/inm.12790

28. Mirzaie A, Halaji M, Dehkordi FS, et al. A narrative literature review on traditional medicine options for treatment of corona virus disease 2019 (COVID-19). Complement Ther Clin Pract. 2020; 40: 101214. Accesible en: https://doi.org/10.1016/j.ctcp.2020.101214

29. Roney LN, Beauvais AM, Bartos S. Igniting Change Supporting the Well-Being of Academicians Who Practice and Teach Critical Care. Critical Care Nursing Clinics of North America. 2020; 32(3): 407-19. Accesible en: https://doi.org/10.1016/j.cnc.2020.05.008

30. Rosa WE, Davidson PM. Coronavirus disease 2019 (COVID-19): strengthening our resolve to achieve universal palliative care. International Nursing Review. 2020; 67(2): 160-3. Accesible en: https://onlinelibrary.wiley.com/doi/epdf/10.1111/inr.12592 
